\title{
Molecular aspects of pancreatic cancer: focus on reprogrammed metabolism in a nutrient-deficient environment and potential therapeutic targets
}

\author{
ROBERT SŁOTWIŃSKI ${ }^{1}$, GUSTAW LECH ${ }^{2}$, SYLWIA MAEGORZATA SŁOTWIŃSKA ${ }^{3}$ \\ ${ }^{1}$ Department of Immunology, Biochemistry and Nutrition, Medical University of Warsaw, Warsaw, Poland \\ ${ }^{2}$ Department of General, Gastroenterological and Oncological Surgery, Medical University of Warsaw, Warsaw, Poland \\ ${ }^{3}$ Department of Oral Hygiene, Medical University of Warsaw, Warsaw, Poland
}

\begin{abstract}
Pancreatic ductal adenocarcinoma (PDAC) is still burdened with high mortality (5-year survival rate $<9 \%$ ) due to late diagnosis, aggressiveness, and a lack of more effective treatment methods. Early diagnosis and new therapeutic approaches based on the reprogrammed metabolism of the tumor in a nutrient-deficient environment are expected to improve the future treatment of PDAC patients. Research results suggest that genetic and metabolic disorders may precede the onset of neoplastic changes, which should allow for earlier appropriate treatment. Glycolysis and glutaminolysis are the most investigated pathways associated with the highest aggressiveness of pancreatic tumors. Blocking of selected metabolic pathways related to the local adaptive metabolic activity of pancreatic cancer cells improving nutrient acquisition and metabolic crosstalk within the microenvironment to sustain proliferation may inhibit cancer development, increase cancer cells death, and increase sensitivity to other forms of treatment (e.g., chemotherapy). Depriving cancer cells of important nutrients (glucose, glutamine) revealed tumor "checkpoints" for the mechanisms that drive cell proliferation and metastasis formation in order to determine its accuracy for individualization of the therapeutic approach. The present review highlights selected metabolic signaling pathways and their regulators aimed at inhibiting the neoplastic process. Particular attention has been paid to the adaptive metabolism of pancreatic cancer, which promotes its development in an oxygen-deficient and nutrient-poor environment.
\end{abstract}

Key words: pancreatic carcinoma, reprogrammed metabolism, therapeutic targets.

(Cent Eur J Immunol 2021; 46 (2): 258-263)

\section{Introduction}

Pancreatic cancer remains the most aggressive cancer, and despite advances in the detection and management, the 5-year survival rate still stands at just 9\%, the primary treatment of which is extensive surgery [1-4]. Surgery, if feasible, prolongs 5-year survival up to approximately 20-30\%; however, this applies to a relatively small group of patients $(20 \%)$ because, at the time of diagnosis, the tumor is unresectable. In patients who undergo a successful potentially curative operation, over $80 \%$ of them eventually develop local recurrence or metastases [5-7]. The limited efficacy of other treatments (e.g., first-line complex chemotherapy - FOLFIRINOX/(5-fluorouracil [5-FU], folinic acid, irinotecan, and oxaliplatin), gemcitabine [GEM] and nab-paclitaxel), especially in patients with metastatic cancer (5-year survival rate of only $2 \%$ ), indicates that pancreatic cancer cells in the majority of treated patients are resistant to this type of therapy [8-10]. According to the APACT trial (updated version 6 January 2020), there was no significant benefit in the median disease-free survival for gemcitabine plus nab-paclitaxel vs. gemcitabine alone in the adjuvant setting of PDAC treatment [11].

Due to the high mortality and limited effectiveness of standard treatments, resulting from the high aggressiveness of PDAC, increasing attention has been paid to the basic molecular mechanisms that enable local tumor development and metastasis formation. Of particular interest, both in diagnostic and therapeutic terms, are the disturbances in signaling pathways associated with important energy sources for pancreatic cancer cells. For example, reprogramming of metabolic signaling pathways regulated by KRAS and TP 53 genes promotes the development of

Correspondence: Prof. Robert Słotwiński, Department of Immunology, Biochemistry and Nutrition, Medical University of Warsaw, Warsaw, Poland, e-mail: robert_slotwinski@yahoo.com

Submitted: 31.03.2021; Accepted: 8.04.2021 
pancreatic cancer by increasing glucose uptake, directing glucose carbon flux into the pentose phosphate pathway (PPP), and increasing the dependence of cancer cells on glutamine [12-14]. Previous studies have shown that increasing glycolysis, enabling cancer cell survival, is one of the hallmarks of pancreatic cancer, and one of the key enzymes involved in glycolysis is pyruvate kinase (PK), the dimeric form of which (PKM2) is released by tumor cells into the peripheral blood, and can be used as a non-specific marker [15, 16]. In studies on pancreatic cancer cell lines (BxPc-3, CFPAC-1, PANC-1), it was found that inhibition of glycolysis in the presence of 2-deoxyglucose (2-DG) increased cancer cell death and their sensitivity to GEM [17]. Other studies demonstrated that low glucose enhanced metformin's inhibitory effect on pancreatic cancer cells (human PANC-1) by suppressing glycolysis and inducing energy stress via upregulation of miR-210-5p [18]. Furthermore, epigallocatechin-3-gallate (EGCG - a major polyphenol from green tea) also affected glycolysis by suppressing the extracellular acidification rate through the reduction of the activity and levels of the glycolytic enzymes phosphofructokinase and pyruvate kinase [19].

The activating mutations in KRAS were found in lowgrade pancreatic intraepithelial neoplasia (PanIN), which could indicate that metabolic reprograming may precede the appearance of the premalignant lesions [20]. An important diagnostic element has emerged from metabolic disorders related to the local adaptive metabolism of pancreatic cancer, which affect the energy and nutritional requirements and cancer aggressiveness, including proliferation rate, metastasis formation, resistance to apoptosis and chemotherapy, glutamine and hypoxia sensitivity, and redox maintenance [21]. Of particular interest is the problem of early identification of the neoplastic cells' microenvironmental requirements at different stages of cancer development based on changes in metabolic signaling pathways' reprograming, as this may lead to a new therapeutic approach. Research results suggest that the development of pancreatic cancer, from the first clinically elusive symptoms through premalignant lesions (PanIN stage I-II) to carcinoma in situ (PanIN stage III) and rapidly developing metastatic tumor, may take even more than 20 years [22-24]. The process of carcinogenesis involves the gradual acquisition of mutations in oncogenes and suppressor genes (KRAS, TP53, SMAD4, CDKN2A-cyclin-dependent kinase inhibitor $2 \mathrm{~A}, \mathrm{p} 16)$ and changes in the micro-environment [25, 26]. Unfortunately, in spite of the long "diagnostic window", pancreatic cancer is usually detected in its advanced stage, and the aggressive course of the neoplastic disease, with rapidly progressing malnutrition, worsens treatment outcomes. The breakdown of proteins throughout the body, associated with malnutrition, may be an early event in the development of PDAC [27]. High levels of branched-chain amino acids were detected in plasma samples associated with pancreatic cancer pa- tients. Recently, significant differences in plasma amino acid concentrations were observed between diagnostic categories (malignant vs. benign lesions and histological cancer types) and PDAC stages [28].

Taking into account the problems with the early diagnosis and treatment of PDAC, the present review highlights selected metabolic signaling pathways and their regulators aimed at inhibiting the neoplastic process. Particular attention has been paid to the adaptive metabolism of pancreatic cancer, which promotes its development in an oxygen-deficient and nutrient-poor environment.

\section{Alterations in the selected metabolic pathways}

Hopes for the improvement of treatment outcomes in PDAC patients are primarily raised by the possibility of inhibiting selected metabolic pathways related to the metabolism of glucose, amino acids, and lipids, which are reprogrammed mainly due to the changes in key enzymes and transporters. Some of them are closely regulated by oncogenic KRAS, which is persistently activated upon mutation in pancreatic cancer (mutationally activated KRAS is present in $>90 \%$ of PDAC and represents the earliest genetic alteration) and has a key role in metabolic reprogramming and particularly in the glycolytic switch [26]. Metabolic reprogramming includes overexpression of glucose transporters and glycolytic enzymes, high-speed adenosine triphosphate (ATP) production, and accumulation of lactate, which contributes to tumor acidosis [29]. The enhanced expression of the glycolytic enzymes is associated with PDAC invasiveness and a bad prognosis [30]. It was discovered that gene-dosage increase of mutant KRAS in human PDAC precursors (PanINs) drives both early tumorigenesis and metastasis, but there are still no KRAS inhibitors that have passed the early phase of clinical trials [31]. Currently, only a small clinical trial has provided supporting evidence to demonstrate the power of KRAS inhibition and the potential for compounds like AMG 510 to be developed further [32].

Unlike healthy cells, cancer is highly glycolytic, even in the presence of oxygen ("aerobic glycolysis", also called the "Warburg effect") and reduced mitochondrial activity. To fuel mitochondrial oxidative phosphorylation (OXPHOS), cancer cells can induce glycolysis in the stromal cells, which helps regulate nutrient availability [33]. On the other hand, a critical enzyme (prolyl 4-hydroxylase subunit alpha-1 - P4HA1) involved in collagen synthesis controls glycolysis through HIF1 $\alpha$ and the P4HA/ HIF1 feedback loop drives the glycolytic and malignant phenotypes of pancreatic cancer [34]. Growing evidence demonstrates that many signal molecules, including oncogenes and tumor suppressors, are involved in the process, but how oncogenic signals attenuate mitochondrial function (reduction of mitochondrial oxidation) and promote 
the switch to glycolysis remains unclear [35]. However, it is known that glycolysis is the main source of energy production (ATP) in hypoxic conditions, which enables faster tumor growth. Dense desmoplastic stroma (taking up to $90 \%$ of the tumor volume) creates ischemia and a hypoxic microenvironment with limited nutrients, which is associated with metabolic stress and cancer cell invasiveness [36]. Oncogenic KRAS upregulates the expression of the glucose transporter (GLUT-1), increasing glucose influx, and of the hexokinase (HK) 1-2 and phosphofructokinase enzymes, which speed up glycolytic activity and production of ATP. Increased GLUT-1 expression has been found in many cancers (e.g., breast, kidney, pancreatic, liver, lung, cervical, ovarian) [37, 38]. High GLUT-1 expression predicted shorter survival in patients with pancreatic cancer. Moreover, GLUT-1 expression was associated with a tumor size of $>2 \mathrm{~cm}$ and the presence of lymph node metastasis [39]. Also, the latest immunohistochemistry studies suggest that GLUT-1 could be related to higher aggressivity in PDAC and could be used as a prognostic marker, identifying patients with a worse response to current therapies who could benefit from more aggressive treatments [40]. Due to the increased glycolytic rate in PDAC, increased expression of lactate transporters (monocarboxylate transporters - MCT1/4) to neutralize intracellular acidification has also been detected, and inhibition or knockdown of MCT resulted in inhibited lactate flux and regulated cancer cell lines' invasiveness [41, 42].

Activation of the transcription of genes encoding glucose transporters and genes encoding glycolytic enzymes is influenced by the ischemia-activated transcription factor HIF1 (hypoxia-inducible factor 1), which plays a key role in reprogramming the metabolism of cancer cells. Hypoxia-inducible factor 1 is the master regulator of glucose metabolism and the induction of mitochondrial autophagy by HIF1 may be another example of an adaptation of cancer cells to hypoxia [43]. HIF1 is over-expressed in pancreatic cancer and associated with poor prognosis [44]. Earlier studies have shown that the disruption of the HIF1 pathway might be effective in the treatment of pancreatic cancers [45]. In such research (in SCID mice), pancreatic cancer cells were sensitive to apoptosis, hypoxia, and glucose deprivation. Targeting HIF1 $\alpha$ or de novo pyrimidine biosynthesis, in combination with gemcitabine, strongly diminishes tumor burden [46]. Reduced expression of genes which regulate flux into pyrimidine biosynthesis (TKT, CTPS), correlates with better prognosis in pancreatic cancer patients on fluoropyrimidine analogs (like 5-FU, a second-line chemotherapy).

Glycolysis and glutaminolysis are the most investigated pathways associated with the highest aggressiveness of pancreatic tumors. The most common signaling molecules involved in these metabolic pathways of PDAC are the phosphatidylinositol 3-kinase (PI3K), protein kinase B (known as AKT), adenosine monophosphate-activated pro- tein kinase (AMPK), and the mammalian target of rapamycin (mTOR) as well as the oncogenic proteins KRAS and MYC (implicated in the induction of glycolysis genes), and the tumor suppressor p53 [47, 48]. Tumor cells have aberrant activation of mTORC 1 that induces an anabolic growth program resulting in nucleotide, protein, and lipid synthesis. In addition to glucose, the main source of energy is glutamine, which is also important in lipid biosynthesis, as a nitrogen donor for nucleotide synthesis, carbon for the mitochondrial tricarboxylic acid (TCA) cycle, and in maintaining redox balance $[49,50]$. These mechanisms lead to the addiction of pancreatic cancer cells to glutamine.

There is a large body of evidence to suggest that oncogenic KRAS and overactivated signaling factors (B-Raf, PI3K, AKT) strongly promote pancreatic cancer [51]. KRAS-driven pancreatic cancer cells were found to be dependent on increased glycolysis, glutamine consumption, the PPP, and autophagy. For example, PI3K signaling (activated by KRAS) plays a central node in the complex cellular signaling networks to impact cancer cell growth, motility, metabolism, and survival [52]. PI3K signaling also modifies the tumor microenvironment to dictate disease outcome, and mutations in the PI3K signaling cascade, accompanied by activation of parallel signaling pathways (AKT, mTOR), make PI3K a promising candidate for drug therapy (e.g., metformin + gemcitabine + erlotinib, MK2206 + fluorouracil + oxaliplatin + selumetinib or capecitabine + cetuximab + everolimus in phase II trials). It has been found that the $\mathrm{PI} 3 \mathrm{~K} / \mathrm{mTORC} 2$ pathway increases GOT1 (glutamic-oxaloacetic transaminase 1 - cytoplasmic form) expression and stimulates non-canonical glutamine metabolism by targeting HIF $2 \alpha$, promoting the progression of PDAC [53]. The results of these studies confirmed that HIF2 $\alpha$ may be a therapeutic target in patients with PDAC. Moreover, the PI3K/AKT pathway has been reported to inhibit cellular apoptosis to stimulate cell proliferation in pancreatic cancer [54]. Small molecule therapeutics that block PI3K signaling can inhibit the neoplastic process by blocking numerous aspects of the tumor cell phenotype [55]. Increased expression of the mitochondrial glutamine transporter (SLC1A5 variant) has recently been found to mediate ATP production and glutathione synthesis, which is induced by glutamine and increases pancreatic cancer cell resistance to gemcitabine [56]. Adenosine monophosphate-activated protein kinase, which is a regulator of glycolysis, also plays an essential role in tumorigenesis. Recent research has revealed that AMPK activation is highly involved in pancreatic cancer progression and exerts its pro-tumorigenic functions partly by sustaining glycolytic activity [57]. Inhibition of AMPK blocked the proliferation, migration, and invasion ability of pancreatic cancer cell lines, and AMPK suppression led to cell cycle arrest and remarkably induced apoptosis. In another study regulating cellular redox, targeting KRAS/ AMPK signaling and reversing metabolic reprogramming 
were suggested as effective approaches to eliminate cancer stem cells (CSCs) and enhance chemosensitivity to GEM to improve the prognosis of pancreatic cancer patients [58].

It is understood that one of the more important features of pancreatic cancer is metabolic rewiring as a prerequisite for tumor initiation and progression, and the targeting of an individual metabolic phenotype with corresponding metabolic inhibitors was considered a promising therapeutic approach. However, molecular changes leading to the metabolic adaptation of pancreatic cancer cells remain unclear. Perhaps, metabolic pathways' intercellular "dialogue" explains how pancreatic cancer cells, despite fuel limitation, maintain their increased proliferation and the ability to form metastases. Of particular interest in the study of tumor aggressiveness is the role of the microenvironment, in which pancreatic cancer cells and the corresponding stroma evolve together to form a dynamic feedback loop that supports cancer metastatic transition. In this process, cancer cells recruit and transform stromal cells, which in turn remodel the extracellular matrix (ECM) of the stroma [59].

\section{Changes of the adaptive metabolic pathways}

More and more data suggest that disruption of the adaptive mechanism may be of benefit in the treatment of pancreatic cancer. An important adaptive mechanism for cancer cell proliferation is the ability to cope with oxidative stress (maintaining redox) in an oxygen-deficient and nutrient-poor environment. Glutamine (Gln), which is a non-essential amino acid for normal cells, plays a key role in maintaining redox homeostasis of cancer cells and, therefore, may be a candidate for therapeutic purposes [60]. Unlike normal cells, pancreatic cancer cells have been shown to follow a distinct metabolic pathway that drives the TCA cycle, in which aspartate-derived Gln is transported into the cytoplasm, where, after conversion to oxaloacetate (OAA) by aspartate transaminase (GOT1), and then into malate and pyruvate, it can increase the NADPH/NADP+ ratio, which maintains the redox state [61]. Normal cells utilize glutamate dehydrogenase (GLUD1) to convert Gln-derived glutamate (Glu) into $\alpha$-ketoglutarate in the mitochondria to fuel the TCA cycle. It has been shown that glutamine deprivation or inhibition of mitochondrial aspartate transaminase (GOT2) may result in suppression of pancreatic cancer growth [62]. In addition, these studies indicated the significant importance of GOT2 in senescence regulation of PDAC, which may have new therapeutic implications. Senescence has been proposed to be an important tumor-suppressive mechanism $[63,64]$ and a selective fibroblast growth factor receptor 4 (FGFR4) inhibitor (BLU9931) reduced PDAC cell proliferation and invasion while promoting their senescence [65]. Targeting FGFR4 overexpressed in half of PDACs in combination with senolysis (e.g., quercetin) could provide a novel therapeutic strategy in pancreatic cancer patients. Interestingly, KRAS knockdown resulted in a marked increase in GLUD1 and a decrease in GOT1 expression at the transcriptional level as well as the protein level in multiple PDAC lines, which indicated that oncogenic KRAS plays a critical role in coordinating the shift in Gln metabolism to maintain tumor growth and survival. KRAS promotes glucose uptake and enhances glycolysis by inducing the expression of GLUT1, the glucose transporter, and other key glycolysis enzymes [66]. Transcriptome and metabolomic analyses indicate that $\mathrm{Kras}^{\mathrm{G} 12 \mathrm{D}}$ serves a vital role in controlling tumor metabolism and channeling of glucose intermediates into the hexosamine biosynthesis and PPP [66]. The essentiality of this pathway in PDAC and the fact that it is dispensable in normal cells may provide novel therapeutic approaches. Disrupting glutamine metabolism by inhibiting glutaminase (GLS), and thus reducing the antioxidant potential of glutathione (GSH) and nicotinamide adenine dinucleotide phosphate (NADPH), leads to reduced proliferation of pancreatic cancer cells. Although the in vitro effects on proliferation were quite marked, these were lost in multiple in vivo models of PDAC [60]. Another adaptive mechanism to metabolic stresses used by PDAC cells to survive the nutrient-poor tumor microenvironment involving accumulation of miR135 in tumor cells in response to glutamine deprivation requires ROS-dependent activation of mutant p53 and should also be recalled [67]. The authors of these studies showed that miR-135 targets phosphofructokinase-1 (PFK1) and inhibits aerobic glycolysis, thereby promoting the utilization of glucose to support the TCA cycle. MiR-135 silencing sensitizes PDAC cells to glutamine deprivation and represses tumor growth in vivo.

\section{Conclusions and future directions}

The short period of survival in patients with PDAC, a problem which continues despite the progress of knowledge, indicates the strong need to verify promising directions of research in terms of their usefulness in diagnostics and treatment. Early diagnosis and new therapeutic approaches based on the adaptive metabolism of the tumor in a nutrient-deficient environment are expected to improve the future treatment of PDAC patients. The present research results suggest that pancreatic cancer is a chronic disease whose development stretches over many years, which should enable earlier detection and more effective treatment. Genetic mutations and metabolic reprogramming may precede the appearance of premalignant lesions (PanIN) and pancreatic tumor formation with metastases. Therapeutic intervention at the stage of metabolic changes that can be inhibited or even reversed may improve treatment outcomes. It was found that blocking selected metabolic pathways related to the local adaptive metabolic activity of pancreatic cancer cells, improving nutrient acquisition and metabolic crosstalk 
within the microenvironment to sustain proliferation, may inhibit cancer development, increase cancer cell death, and increase sensitivity to other forms of treatment (e.g., chemotherapy). Research aimed at "starving" cancer cells by depriving them of their basic energy sources (ATP) allows for a better understanding of the possibilities of regulating the principal metabolic reprogramming found in pancreatic cancer. Based on a better understanding of the diverse functions of pancreatic cancer signaling proteins (e.g., glucose transporters, glycolytic enzymes), critical points for alternative therapy and prognostic markers have been established to identify patients with a worse response to current therapy. It should be emphasized that the results of basic research collected in this review shed new light on the reasons for the reprogramming of metabolic signaling pathways in pancreatic cancer (including cancer cell malnutrition, sensitivity to nutrient deprivation, and addiction of pancreatic cancer cells to certain nutrients such as glutamine), which can be used for early diagnosis and may also carry prognostic value so, in the future, it will allow the implementation of novel, more effective therapy. Moreover, it was understood that the heterogeneous nature of pancreatic cancer may be reflected in metabolic reprogramming, which, for example, in the glycolytic cancer subtype is associated with greater aggressiveness and resistance to chemotherapy. Thus, employing metabolism to improve the personalized approach in the diagnosis and treatment of pancreatic cancer is still a promising direction of research.

\section{The authors declare no conflict of interest.}

\section{References}

1. Siegel RL, Miller KD, Jemal A (2020): Cancer statistics 2020. CA Cancer J Clin 70: 7-30.

2. Latenstein AEJ, Geest LGM, Bonsing BA, et al. (2020): Nationwide trends in incidence, treatment and survival of pancreatic ductal adenocarcinoma. Eur J Cancer 125: 83-93.

3. Neuzillet C, Tijeras-Raballand A, Bourget P, et al. (2015): State of the art and future directions of pancreatic ductal adenocarcinoma therapy. Pharmacol Ther 155: 80-104.

4. Rawla P, Sunkara T, Gaduputi V (2019): Epidemiology of pancreatic cancer: global trends, etiology and risk factors. World J Oncol 10: 10-27.

5. Huang L, Jansen L, Balavarca Y, et al. (2019): Resection of pancreatic cancer in Europe and USA: an international largescale study highlighting large variations. Gut 68: 130-139.

6. Huang L, Jansen L, Balavarca Y, et al. (2018): Stratified survival of resected and overall pancreatic cancer patients in Europe and the USA in the early twenty-first century: a large, international population-based study. BMC Medicine 16: 125 (http://creativecommons.org/licenses/by/4.0/)

7. Kleeff J, Reiser C, Hinz U, et al. (2007) Surgery for recurrent pancreatic ductal adenocarcinoma. Ann Surg 245: 566-572.

8. Conroy T, Desseigne F, Ychou M, et al. (2011): The Groupe Tumours Digestives of Unicancer and the PRODIGE Intergroup. FOLFIRINOX versus gemcitabine for metastatic pancreatic cancer. N Engl J Med 364: 1817-1825.
9. Burris HA III, Moore MJ, Andersen J, et al. (1997): Improvements in survival and clinical benefit with gemcitabine as first line therapy for patients with advanced pancreas cancer: a randomized trial. J Clin Oncol 15: 2403-2413.

10. Moore MJ, Goldstein D, Hamm J, et al. (2007): Erlotinib plus gemcitabine compared with gemcitabine alone in patients with advanced pancreatic cancer: a phase III trial of the $\mathrm{Na}$ tional Cancer Institute of Canada Clinical Trials Group. J Clin Oncol 25: 1960-1966.

11. Reni M, Riess H, O'Reilly EM, et al. (2020): Phase III APACT trial of adjuvant nab-paclitaxel plus gemcitabine (nab-P + Gem) versus gemcitabine (Gem) alone for patients with resected pancreatic cancer (PC): Outcomes by geographic region. J Clin Oncol 38: 15 suppl.: 4515-4515 (https://clinicaltrials.gov/ct2/show/results/NCT01964430?view=results).

12. Son J, Lyssiotis CA, Ying H, et al. (2013): Glutamine supports pancreatic cancer growth through a KRAS-regulated metabolic pathway. Nature 496: 101-105.

13. Zhang XD, Qin ZH, Wang J (2010): The role of p53 in cell metabolism. Acta Pharmacol Sin 31: 1208-1212.

14. Abrego J, Gunda V, Vernucci E, et al. (2017): GOT1-mediated anaplerotic glutamine metabolism regulates chronic acidosis stress in pancreatic cancer cells. Cancer Lett 400: 37-46.

15. Liberti MV, Locasale JW (2016): The Warburg effect: how does it benefit cancer cells? Trends Biochem Sci 41: 211-218.

16. Ventrucci M, Cipolla A, Racchini C, et al. (2004): Tumor M2-pyruvate kinase, a new metabolic marker for pancreatic cancer. Dig Dis Sci 49: 1149-1155.

17. Dai S, Penga Y, Zhu Y, et al. (2020): Glycolysis promotes the progression of pancreatic cancer and reduces cancer cell sensitivity to gemcitabine. Biomed Pharmacother 121: 109521.

18. Ma M, Ma C, Li P, et al. (2020): Low glucose enhanced metformin's inhibitory effect on pancreatic cancer cells by suppressing glycolysis and inducing energy stress via upregulation of miR-210-5p. Cell Cycle 19: 2168-2181.

19. Wei R, Hackman RM, Wang Y, Mackenzie GG (2019): Targeting glycolysis with epigallocatechin-3-gallate enhances the efficacy of chemotherapeutics in pancreatic cancer cells and xenografts. Cancers 11: 1496.

20. Takai E, Yachida S (2015): Genomic alterations in pancreatic cancer and their relevance to therapy. World J Gastrointest Oncol 7: 250-258.

21. Biancur DE, Kimmelman AC (2018): The plasticity of pancreatic cancer metabolism in tumor progression and therapeutic resistance. Biochim Biophys Acta Rev Cancer 1870: 67-75.

22. Yachida S, Jones S, Bozic I, et al. (2010): Distant metastasis occurs late during the genetic evolution of pancreatic cancer. Nature 467: 1114-1117.

23. Storz P, Crawford HC (2020): Carcinogenesis of pancreatic ductal adenocarcinoma. Gastroenterology 158: 2072-2081.

24. Brosens LAA, Hackeng WM, Offerhaus GJ, et al. (2015): Pancreatic adenocarcinoma pathology: changing "landscape". J Gastrointest Oncol 6: 358-374.

25. Aguirre AJ, Nowak JA, Camarda ND, et al. (2018): Real-time genomic characterization of advanced pancreatic cancer to enable precision medicine. Cancer Discov 8: 1096-1111.

26. Jones S, Zhang X, Parsons DW, et al. (2008): Core signalling pathways in human pancreatic cancers revealed by global genomic analyses. Science 321: 1801-1806.

27. Mayers JR, Wu C, Clish CB, et al. (2014): Elevation of circulating branched-chain amino acids is an early event in human pancreatic adenocarcinoma development. Nat Med 20: 1193-1198.

28. Tumas J, Baskirova I, Petrenas T, et al. (2019): Towards a personalized approach in pancreatic cancer diagnostics 
through plasma amino acid analysis. Anticancer Res 39: 2035-2042.

29. Vaupel P, Schmidberger H, Mayer A (2019): The Warburg effect: essential part of metabolic reprogramming and central contributor to cancer progression. Int J Radiat Biol 95: 912-919.

30. Tian G, Li G, Liu P, et al. (2020): Glycolysis-based genes associated with the clinical outcome of pancreatic ductal adenocarcinoma identified by the cancer genome atlas data analysis. DNA Cell Biol 39: 417-427.

31. Mueller S, Engleitner T, Maresch R, et al. (2018): Evolutionary routes and KRAS dosage define pancreatic cancer phenotypes. Nature 554: 62-68.

32. Canon J, Rex K, Saiki AY, et al. (2019): The clinical KRAS (G12C) inhibitor AMG 510 drives anti-tumour immunity. Nature 575: 217-223.

33. Wilde L, Roche M, Domingo-Vidal M, et al. (2017): Metabolic coupling and the reverse Warburg effect in cancer: implications for novel biomarker and anticancer agent development. Semin Oncol 44: 198-203.

34. Cao X, Cao Y, Li W, et al. (2019): P4HA1/HIF1 feedback loop drives the glycolytic and malignant phenotypes of pancreatic cancer. Biochem Biophys Res Commun 516: 606-612.

35. Yu L, Chen X, Sun X, et al. (2017): The glycolytic switch in tumors: how many players are involved? J Cancer 8: 3430-3440.

36. Kamphorst JJ, Cross JR, Fan J, et al. (2013): Hypoxic and Ras-transformed cells support growth by scavenging unsaturated fatty acids from lysophospholipids. Proc Natl Acad Sci USA 110: 8882-8887.

37. Airley RE, Mobasheri A (2007): Hypoxic regulation of glucose transport, anaerobic metabolism and angiogenesis in cancer: novel pathways and targets for anticancer therapeutics. Chemotherapy 53: 233-256.

38. Macheda ML, Rogers S, Best JD (2005): Molecular and cellular regulation of glucose transporter (GLUT) proteins in cancer. J Cell Physiol 202: 654-662.

39. Sharen G, Peng Y, Cheng H, et al. (2017): Prognostic value of GLUT-1 expression in pancreatic cancer: Results from 538 patients. Oncotarget 8: 19760-19767.

40. Boira MA, Di Martino M, Gordillo C, et al. (2020): GLUT-1 as a predictor of worse prognosis in pancreatic adenocarcinoma: immunohistochemistry study showing the correlation between expression and survival. BMC Cancer 20: 909.

41. Cameron ME, Yakovenko A, Trevino JG (2018): Glucose and lactate transport in pancreatic cancer: glycolytic metabolism revisited. J Oncol 2018: 6214838.

42. Kong SC, Nřhr-Nielsen A, Zeeberg K, et al. (2016): Monocarboxylate transporters MCT1 and MCT4 regulate migration and invasion of pancreatic ductal adenocarcinoma cells. Pancreas 45: 1036-1047.

43. Semenza GL (2010): Defining the role of hypoxia-inducible factor 1 in cancer biology and therapeutics. Oncogene 29: 625-634.

44. Hao J (2015): HIF-1 is a critical target of pancreatic cancer. Oncoimmunology 4: e1026535.

45. Chen J, Zhao S, Nakada K, et al. (2003): Dominant-negative hypoxia-inducible factor- $1 \alpha$ reduces tumorigenicity of pancreatic cancer cells through the suppression of glucose metabolism. Am J Pathol 162: 1283-1291.

46. Shukla SK, Purohit V, Mehla K, et al. (2017): MUC1 and HIF-1alpha signaling crosstalk induces anabolic glucose metabolism to impart gemcitabine resistance to pancreatic cancer. Cancer Cell 32: 71-87.

47. DeBerardinis RJ, Chandel NS (2016): Fundamentals of cancer metabolism. Sci Adv 2: e1600200.
48. Cairns RA, Harris IS, Mak TW (2011): Regulation of cancer cell metabolism. Nat Rev Cancer 11: 85-95.

49. Liang C, Qin Y, Zhang B, et al. (2016): Energy sources identify metabolic phenotypes in pancreatic cancer. Acta Biochim Biophys Sin 48: 969-979.

50. Halbrook CJ, Lyssiotis CA (2017): Employing metabolism to improve the diagnosis and treatment of pancreatic cancer. Cancer Cell 31: 5-19.

51. Blum R, Kloog Y (2014): Metabolism addiction in pancreatic cancer. Cell Death Dis 5: e1065.

52. Murthy D, Attri KS, Singh PK (2018): Phosphoinositide 3-kinase signaling pathway in pancreatic ductal adenocarcinoma progression, pathogenesis, and therapeutics. Front Physiol 9: 335.

53. Li W, Chen C, Zhao X, et al. (2017): HIF-2a regulates non-canonical glutamine metabolism via activation of PI3K/ mTORC2 pathway in human pancreatic ductal adenocarcinoma. J Cell Mol Med 21: 2896-2908.

54. Mao Y, Xi L, Li Q, et al. (2016): Regulation of cell apoptosis and proliferation in pancreatic cancer through PI3K/Akt pathway via Polo-like kinase 1. Oncol Rep 36: 49-56.

55. Akinleye A, Avvaru P, Furqan M, et al. (2013): Phosphatidylinositol 3-kinase (PI3K) inhibitors as cancer therapeutics. J Hematol Oncol 6: 88.

56. Yoo HC, Park SJ, Nam M, et al. (2020): A variant of SLC1A5 is a mitochondrial glutamine transporter for metabolic reprogramming in cancer cells. Cell Metab 31: 267-283.

57. Hu M, Chen X, Ma L, et al. (2019): AMPK inhibition suppresses the malignant phenotype of pancreatic cancer cells in part by attenuating aerobic glycolysis. J Cancer 10: 18701878.

58. Zhao H, Wu S, Li H, et al. (2019): ROS/KRAS/AMPK signaling contributes to gemcitabine-induced stem-like cell properties in pancreatic cancer. Mol Ther Oncolytics 14: 299-312.

59. Emon B, Bauer J, Jain Y, et al. (2018): Biophysics of tumor microenvironment and cancer metastasis - a mini review. Comput Struct Biotechnol J 16: 279-287.

60. Biancur DE, Paulo JA, Małachowska B, et al. (2017): Compensatory metabolic networks in pancreatic cancers upon perturbation of glutamine metabolism. Nat Commun 8: 15965.

61. Son J, Lyssiotis CA, Ying H, et al. (2013): Glutamine supports pancreatic cancer growth through a Kras-regulated metabolic pathway. Nature 496: 101-105.

62. Yang S, Hwang S, Kim M, et al. (2018): Mitochondrial glutamine metabolism via GOT2 supports pancreatic cancer growth through senescence inhibition. Cell Death Dis 9: 55.

63. Nardella C, Clohessy JG, Alimonti A, Pandolfi PP (2011): Pro-senescence therapy for cancer treatment. Nat Rev Cancer 11: 503-511.

64. Collado M, Serrano M (2010): Senescence in tumours: evidence from mice and humans. Nat Rev Cancer 10: 51-57.

65. Sasaki N, Gomi F, Yoshimura H, et al. (2020): FGFR4 inhibitor BLU9931 attenuates pancreatic cancer cell proliferation and invasion while inducing senescence: evidence for senolytic therapy potential in pancreatic cancer. Cancers 12: 2976.

66. Ying H, Kimmelman AC, Lyssiotis CA, et al. (2012): Oncogenic Kras maintains pancreatic tumors through regulation of anabolic glucose metabolism. Cell 149: 656-670.

67. Yang Y, Gabra MBI, Hanse EA, et al. (2020): MiR-135 suppresses glycolysis and promotes pancreatic cancer cell adaptation to metabolic stress by targeting phosphofructokinase-1. Nat Commun 10: 809. 